\title{
Potential impact of
}

\section{cytochrome P450 3A5 in} human liver on drug

\section{interactions with triazoles}

\section{Hiroshi Yamazaki, Minako Nakamoto, Makiko Shimizu,} Norie Murayama \& Toshiro Niwa

Laboratory of Drug Metabolism and Pharmacokinetics, Showa Pharmaceutical University, Machida, Tokyo 194-8543, Japan

\section{Correspondence}

Professor Hiroshi Yamazaki PhD, Showa

Pharmaceutical University, 3-3165

Higashi-tamagawa Gakuen, Machida,

Tokyo 194-8543, Japan.

Tel:/Fax: +8142721 1406

E-mail:hyamazak@ac.shoyaku.ac.jp

\section{Keywords}

CYP3A5, CYP3A4, drug interaction, genetic polymorphism, thalidomide, voriconazole

Received

30 November 2009

Accepted

28 January 2010

Cytochrome P450 3A is the main enzyme subfamily involved in the metabolism of a variety of marketed medicines. It is generally believed that the substrate specificity of polymorphic P450 3A5 is similar to that of the predominant P450 3A4 isoform, although some differences in catalytic properties have been found. It has been hypothesized that individuals with CYP3A5*1 (P450 $3 A 5$ expresser) might clear the HIV protease inhibitor saquinavir, administered by mouth, more rapidly than subjects lacking functional CYP3A5 alleles. Enhanced midazolam hydroxylation and cyclosporin metabolism occur in an in vitro P450 3A5 system and in liver microsomes expressing P450 3A5 in the presence of thalidomide. However, inhibition constants $\left(K_{\mathrm{i}}\right)$ of three triazole anti-fungal drugs (itraconazole, fluconazole, and voriconazole) for liver microsomal P450 3A5 are higher than for liver microsomal P450 3A4. To predict drug interactions in vivo, we estimated increases of areas under the curves (AUC) dependent on polymorphic P450 $3 A 5$ expression, using both $1+$ [Inhibitor] / $\mathrm{K}_{\mathrm{i}}$ (recommended in US FDA guidance), and $1+$ [Inhibitor] unbound / $\mathrm{K}_{\mathrm{i}}$ (as recommended by Japanese MHLW Notice). Voriconazole would be expected to cause approximately a three-fold higher increase in AUC in subjects with CYP3A5*3/*3 than in those with $C Y P 3 A 5^{*} 1{ }^{*} 3$, especially when estimated using the FDA guidance. We conclude that drug interactions between marketed drugs may differ substantially between individuals with genetically distinct P450 3A5 catalytic functions.

\section{Introduction}

Cytochrome P450 enzymes (P450s) are heme-thiolate mono-oxygenases involved in oxidation of many endogenous and exogenous substrates [1]. P450 3A is the most important human P450 subfamily due to its high relative abundance in the liver and broad substrate specificity [2, 3]. P450 3A4 is a predominant form generally expressed in the human liver and intestine, whereas P450 3A5, which is expressed polymorphically, might contribute as much as $50 \%$ of hepatic P450 3A in a third to one half of Caucasians and African-Americans [4]. We reported a high frequency of hepatic P450 3A5 expression in a Japanese population $[5,6]$. Despite $83 \%$ homology between P450 3A5 and P450 $3 A 4$, differences in catalytic properties have been demonstrated $[7,8]$.

Triazole antifungal drugs are clinically important for serious, invasive fungal infections [9]. Itraconazole is a fatsoluble synthetic triazole antifungal drug and is a broad spectrum antifungal. Voriconazole was approved in Europe and the United States in 2002 for the treatment of invasive Aspergillosis and has an extremely broad spec- trum. Voriconazole is structurally related to fluconazole, but its activity and handling by the body are completely different. These triazole antifungals cause clinically important drug interactions via their effects on human P450 3A enzymes. This has been studied in vitro and in vivo [10, 11]. However, few reports compare in detail their effects on the kinetics of drug oxidation by P450 3A4 and P450 $3 \mathrm{~A} 5$ or compare their potential for drug interactions resulting from their inhibition of P450 3A4 vs. P450 3A5 [12].

The present paper summarizes the roles of P450 3A enzymes in metabolic clearance and inhibition of triazole antifungal drugs, including voriconazole, emphasising polymorphic P450 3A5 expression in genotyped livers and the potential impact of this on drug interactions and activation (co-operativity).

\section{Methods}

The kinetic parameters used in this study were taken from our earlier reviews $[12,13]$ as well as from newer literature 


\section{Table 1}

Reported inhibition constants of fluconazole and itraconazole toward recombinant P450 3A4- and P450 3A5-mediated midazolam 1'-hydroxylation activities

\begin{tabular}{|c|c|c|c|c|}
\hline Triazole & Inhibition type & $\begin{array}{l}K_{\mathrm{i}}(\mu \mathrm{M}) \\
\text { P450 3A4 }\end{array}$ & P450 3 A5 & References \\
\hline Itraconazole & Competitive & $0.0157(0.0013)$ & - & [22] \\
\hline Fluconazole & Noncompetitive & $9.2 \pm 0.5$ & $85 \pm 3$ & [17] \\
\hline Fluconazole & $\begin{array}{l}\text { Mixed-type (3A4) } \\
\text { competitive (3A5) }\end{array}$ & $7.4 \pm 1.9$ & $53 \pm 4$ & [18] \\
\hline
\end{tabular}

The value in brackets shows $K_{i, f u}$, mic where fu, mic is the unbound free fraction $(8.8 \pm 3.2 \%)$ in the microsomes expressing P450 $3 A 4$.

\section{Table 2}

Reported inhibition constants of fluconazole and itraconazole toward human liver microsomal midazolam 1'-hydroxylation activities

\begin{tabular}{|c|c|c|c|c|}
\hline Inhibitor & Inhibition type & $K_{\mathrm{i}}(\mu \mathrm{M})$ & $\mathrm{IC}_{50}(\mu \mathrm{M})$ & References \\
\hline Itraconazole & - & $0.50 \pm 0.04$ & - & [20] \\
\hline Itraconazole & - & - & 0.15 & [21] \\
\hline Fluconazole & Noncompetitive & $15 \pm 0.8$ & - & In P450 3A5 non-expressing liver microsomes [18]. \\
\hline Fluconazole & Noncompetitive & $25 \pm 15$ & - & In P450 3A5 expressing liver microsomes [18]. \\
\hline Fluconazole & - & $11.9 \pm 0.9$ & - & [20] \\
\hline Fluconazole & - & - & $43 \pm 6$ & [19] \\
\hline
\end{tabular}

The value in brackets shows $I C_{50, f u}$, mic where fu, mic is the free fraction $(19.6 \pm 9.2 \%)$ in human liver microsomes.

and from some original data. Human P450 3A4 and $3 \mathrm{~A} 5$ expressed in Escherichia coli membranes and liver microsomes genotyped for the CYP $3 A 5^{*} 1$ and $3 A 5^{*} 3$ were used as enzyme sources [14]. Inhibition constants $\left(K_{\mathrm{i}}\right)$ of itraconazole, fluconazole, and voriconazole were determined against midazolam hydroxylation catalyzed by liver microsomal P450 3A enzymes [14]. Inhibitory effects of voriconazole on cyclosporin oxidation were also determined [14]. To predict the potential for drug interactions in vivo, increases of areas under the curves (AUC) dependent on polymorphic $\mathrm{P} 4503 \mathrm{~A} 5$ expression were estimated both from $1+[\mathrm{l}] / K_{\mathrm{i}}$ (as recommended in US FDA guidance) and from $1+[1]$ unbound $/ K_{\mathrm{i}}$ (as recommended by Japanese MHLW Notice), where [I] and [I] $]_{\text {unbound }}$ are the total and unbound inhibitor concentrations, respectively, and $K_{\mathrm{i}}$ is the inhibition constant $[11,15]$. Docking simulations of voriconazole and other substrates in a reported structure of P450 3A4 (PDB 1WOG) and a model of P450 3A5 were calculated using MOE software $[14,16]$.

\section{Results of data review and discussion}

Reported inhibition constants $\left(K_{\mathrm{i}}\right)$ of triazoles toward recombinant P450 3A4 and P450 3A5 are summarized in
Table 1. Inhibition by triazoles of 1'-hydroxylation of midazolam varied by a factor of 10 between the $K_{\mathrm{i}}$ values of fluconazole toward P450 3A4 and P450 3A5 [17, 18]. With human liver microsomes as the enzyme source (Table 2 ), reported $I C_{50}$ or $K_{\mathrm{i}}$ values of itraconazole indicated 17 -fold differences [19-22]. Similar results have been obtained with fluconazole [18-20,23, 24]. Apparent differences in $K_{\mathrm{i}}$ of fluconazole were reported between liver microsomes that either do or do not express P450 3A5 [18].

To investigate the apparently low potential for inhibition of triazoles toward P450 3A5 compared with P450 $3 \mathrm{~A} 4$, we conducted inhibition studies with P450 $3 \mathrm{~A} 5$ and P450 3A4 under identical conditions using P450 isoforms expressed in bacterial membranes. Recombinant P450 3A5 catalyzed midazolam 1'-hydroxylation more efficiently than did P450 3A4 (Table 3). $K_{\mathrm{i}}$ values of these triazoles for midazolam hydroxylation catalyzed by recombinant $\mathrm{P} 450$ $3 \mathrm{~A} 4$ were lower than those catalyzed by P450 3 A5. $K_{i}$ values of each of the three triazoles determined using human liver microsomes genotyped for the CYP3A5*3/*3 (poor P450 3A5 expresser) were approximately half those determined using human liver microsomes from the CYP3A5*1/*3 genotype (P450 3A5 expressers) (Table 3 ). It should be mentioned that the apparent kinetic parameters for liver microsomes shown in Table 3 were affected by liver microsomal P450 3A4 and/or P450 3A5. 
Table 3

Examined inhibition constant $\left(K_{\mathrm{i}}\right)$ of itraconazole, fluconazole, and voriconazole on midazolam 1'-hydroxylation activities catalyzed by P450 $3 \mathrm{~A} 4$ and P450 $3 \mathrm{~A} 5$ and human liver microsomes genotyped for the CYP3A5 gene

\begin{tabular}{|c|c|c|c|c|c|}
\hline & $V_{\max }$ & $K_{\mathrm{m}}(\mu \mathrm{M})$ & $\begin{array}{l}K_{\mathrm{i}}(\mu \mathrm{M}) \\
\text { Itraconazole }\end{array}$ & Fluconazole & Voriconazole \\
\hline \multicolumn{6}{|l|}{ Recombinant } \\
\hline \multicolumn{6}{|c|}{ Liver microsomes } \\
\hline $3 A 5 * 3 / * 3$ & $2.0 \pm 0.1^{b}$ & $5 \pm 2$ & $0.13 \pm 0.030$ & $5.1 \pm 1.1$ & $0.20 \pm 0.12$ \\
\hline $3 A 5 * 1 / * 3$ & $1.5 \pm 0.1$ & $2 \pm 1$ & $0.26 \pm 0.032$ & $7.4 \pm 4.2$ & $0.45 \pm 0.12$ \\
\hline
\end{tabular}

${ }^{a} \mathrm{nmol} \mathrm{min}{ }^{-1} \mathrm{nmol}^{-1} \mathrm{P} 4503 \mathrm{~A},{ }^{b} \mathrm{nmol} \mathrm{min}{ }^{-1} \mathrm{mg}^{-1}$ protein. Kinetic parameters are mean and SE by nonlinear regression analysis. $K_{\mathrm{i}}$ values were determined in a competitive manner. The liver microsomal samples genotyped as $3 \mathrm{~A}^{*} 3 /{ }^{*} 3$ and3A5* $1 /{ }^{*} 3$ contained 18 pmol P450 $3 \mathrm{~A} 4$ and 6.1 pmol P450 3 A5 mg ${ }^{-1}$ protein and 17 pmol P450 3 A4 and 24 pmol P450 $3 \mathrm{~A} 5 \mathrm{mg}^{-1}$ protein, respectively $[5,6]$.

\section{Table 4}

Effects of voriconazole on cyclosporin A oxidation activities of recombinant P450 3A4 and P450 3A5 and human liver microsomes genotyped for the CYP3A5 gene

\begin{tabular}{|c|c|c|}
\hline P450 & Control & Plus voriconazole \\
\hline Recombinant & $\left(\mathrm{nmol} \mathrm{min} \mathrm{mmol}^{-1} \mathrm{P} 450\right)$ & \\
\hline P450 3A4 & $0.47(100)$ & $0.18(38)$ \\
\hline P450 3A5 & $0.40(100)$ & $0.27(68)$ \\
\hline Liver microsomes & (nmol $\mathrm{min}^{-1} \mathrm{mg}^{-1}$ protein) & \\
\hline CYP $3 A 5 * 3 / * 3$ & $0.31(100)$ & $0.17(55)$ \\
\hline$C Y P 3 A 5 * 1 / * 3$ & $0.25(100)$ & $0.22(88)$ \\
\hline
\end{tabular}

Cyclosporin A $(10 \mu \mathrm{M})$ was incubated with voriconazole $(0.20 \mu \mathrm{M})$. Data are average from duplicate determinations. Numbers in parentheses are \% of the control. The P450 3A contents in human liver microsomes are shown in the legend of Table 3.

Voriconazole is of particular interest because of different metabolic pathways catalyzed by P450 2C19 and P450 3 A4 [16]. Voriconazole $\mathrm{N}$-oxidation and 4-hydroxylation are catalyzed more efficiently by P450 3A4 than by P450 3A5 [16]. Using voriconazole to inhibit cyclosporin oxidation, it was found that voriconazole was a weak inhibitor of P450 3A5. Liver microsomes expressing P450 3A5 were less inhibited than were microsomes expressing P450 3A4 (Table 4). $V_{\max }$ and $K_{m}$ for tacrolimus metabolism have

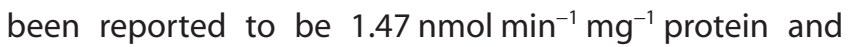
$10.6 \mu \mathrm{moll}^{-1}$ in the absence and $0.37 \mathrm{nmol} \mathrm{min}^{-1} \mathrm{mg}^{-1}$ protein and $5.43 \mu_{\mathrm{mol} \mathrm{I}}^{-1}$ in the presence of voriconazole $\left(200 \mu \mathrm{g} \mathrm{ml}^{-1}=573 \mu \mathrm{mol} \mathrm{I}^{-1}\right)$, respectively, suggesting that voriconazole inhibits both by competitive and noncompetitive mechanisms [25]. Docking simulation revealed that voriconazole can adopt an orientation suitable for $N$-oxidation over the P450 3A4 heme (Figure 1A). However, voriconazole could adopt far from the P450 3A5 heme (Figure 1B), resulting in a slower metabolic rate by $\mathrm{P} 450$ 3 A5 than P450 3A4 in a Michaels-Menten kinetic manner.
Midazolam can adopt an orientation suitable for 1 '-hydroxylation over the P450 3A5 heme (Figure 1C) as well as the P450 3A4 heme. It is noteworthy that thalidomide adapts closely to the P450 3A5 heme (Figure 1D) and enhances midazolam 1'-hydroxylation or cyclosporin $A$ clearance of P450 3A5 [14] via an unexpected phenomenon, which is called activation or heterotropic co-operativity [13], in a mechanism distinct from that of voriconazole. In our preliminary experiments, thalidomide was hydroxylated in a sigmoidal velocity curve vs. substrate, presumably because of docking two substrate molecules into the P450 3A5 pocket, which is called as homotropic co-operativity [13]. Why the mechanism is different between voriconazole and thalidomide is not known but it might be due to substrate molecular size, polarity or affinity towards a P450 3A5 pocket.

It is generally believed that the substrate specificity of polymorphic P450 3A5 is similar to that of the predominant P450 isoform, P450 3A4, although some differences in catalytic properties have been found. It has been hypothesized that individuals with CYP3A5*1 (P450 3A5 expresser) might have a higher clearance of the HIV protease inhibitor saquinavir, administered by mouth, compared with subjects lacking functional CYP3A5 alleles [12]. This and the results summarized in Tables 3 and 4, suggest that inter-individual differences in drug clearances and drug-interactions of triazole antifungal drugs, including voriconazole, are appreciably affected by the CYP3A5 genotypes (Tables 2, 3). Polymorphic P450 3A5 expression in human livers could influence several clinically important drug interactions. To investigate this we estimated the potential for in vivo interaction from the $[I] / K_{\mathrm{i}}$ or $[\mathrm{I}]_{\text {unbound }}: K_{\mathrm{i}}$ ratios, using blood concentration data in their package inserts. In order to calculate the $[\mathrm{I}]_{\text {unbound }}$ : $K_{\mathrm{i}}$ ratios, the pharmacokinetic parameters of each drug were obtained from the literature, together with the $K_{\mathrm{i}}$ values from in vitro studies using human liver microsomes (Table 3 ). The findings indicated that the highest increased AUC caused by 
A

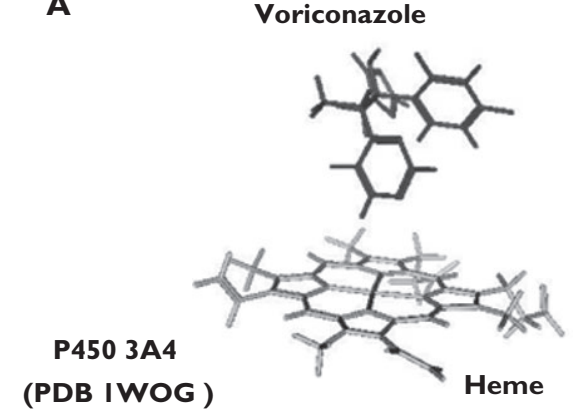

C

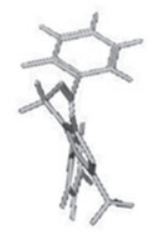

Midazolam

P450 3A5

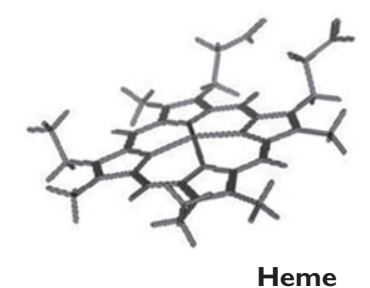

Voriconazole

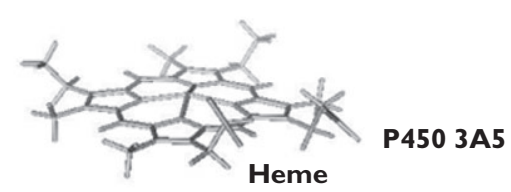

D

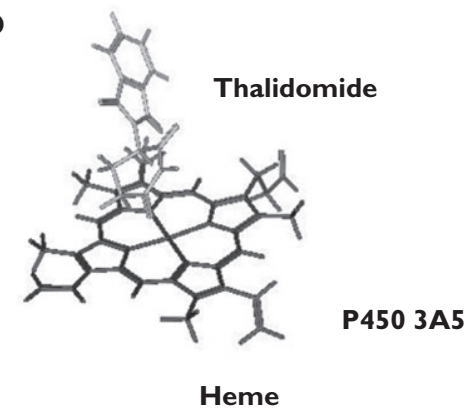

\section{Figure 1}

Docking simulation of voriconazole into a reported structure of P450 3A4 (A) and a homology model of P450 3 A5 (B) in comparison with the cases of midazolam (C) and thalidomide (D). Voriconazole adopted an orientation suitable for P450 3A4 (corresponding to 1 WOG) $(U=21.4)[16]$. A homology model of P450 3A5 was constructed as reported previously [14]

\section{Table 5}

In vivo estimated fold increase in AUC in the presence of itraconazole, fluconazole, and voriconazole from $1+[1] / K_{\mathrm{i}}$ equation in in vitro experiments

\begin{tabular}{|c|c|c|c|c|}
\hline Azoles, single dose ${ }^{a}$ & $\begin{array}{l}\text { Reported maximal plasma } \\
\text { concentration }(\mu \mathrm{M})^{\mathrm{a}}\end{array}$ & fu (\%) & $\begin{array}{l}\text { Fold increase in AUC } \\
3 A 5^{*} 3 / * 3\end{array}$ & $3 A 5 * 1 / * 3$ \\
\hline Itraconazole $200 \mathrm{mg}$ & 0.31 & 0.2 & $3.4(1.0)$ & $2.2(1.0)$ \\
\hline Voriconazole $400 \mathrm{mg}$ & 8.3 & 42 & $43(18)$ & $19(8.7)$ \\
\hline
\end{tabular}

${ }^{a}$ Taken from data in package insert for each medicine. ${ }^{b} K_{i}(\mu \mathrm{M})$ values were taken from Table 3 in human liver microsomes genotyped for the CYP3A5 gene. Numbers in parentheses indicate fold increase in AUC by $1+[I]$ unbound $/ K_{i}$ equation.

co-administered voriconazole would be expected to be low in expressers of P450 3A5 (Table 5). There are not any published data in our literature search so far suggesting/ indicating the changes in vivo shown in Table 5 from the viewpoint of the CYP3A5 genotype. However, drug interactions via P450 3A inhibition should be anticipated especially in subjects of the CYP3A5*3/*3 genotype, who are expected to be poor expressers of P450 3 A5.

In conclusion, CYP3A5 genotype may influence interindividual differences in triazole antifungal drug clearance and the intensity of drug interactions with voriconazole. P450 3A5 also shows the heterotropic co-operativity phenomenon in some cases. Increased AUC caused by co-administered voriconazole should be anticipated as a potential cause of toxicity, especially in subjects with the CYP $3 A 5^{*} 3 * 3$ genotype.

\section{Competing interests}

There are no competing interests to declare. 


\section{REFERENCES}

1 Guengerich FP. Cytochrome P450 and chemical toxicology. Chem Res Toxicol 2008; 21: 70-83.

2 Shimada T, Yamazaki H, Mimura M, Inui Y, Guengerich FP. Interindividual variations in human liver cytochrome P-450 enzymes involved in the oxidation of drugs, carcinogens and toxic chemicals: studies with liver microsomes of 30 Japanese and 30 Caucasians. J Pharmacol Exp Ther 1994; 270: 414-23.

3 Wilkinson GR. Cytochrome P4503A (CYP3A) metabolism: prediction of in vivo activity in humans. J Pharmacokin Biopharm 1996; 24: 475-90.

4 Kuehl P, Zhang J, Lin Y, Lamba J, Assem M, Schuetz J, Watkins PB, Daly A, Wrighton SA, Hall SD, Maurel P, Relling M, Brimer C, Yasuda K, Venkataramanan R, Strom S, Thummel K, Boguski MS, Schuetz E. Sequence diversity in CYP3A promoters and characterization of the genetic basis of polymorphic CYP3A5 expression. Nat Genet 2001; 27: 383-91.

5 Yamaori S, Yamazaki H, Iwano S, Kiyotani K, Matsumura K, Saito T, Parkinson A, Nakagawa K, Kamataki T. Ethnic differences between Japanese and Caucasians in the expression levels of mRNAs for CYP3A4, CYP3A5 and CYP3A7: lack of co-regulation of the expression of CYP3A in Japanese livers. Xenobiotica 2005; 35: 69-83.

6 Yamaori S, Yamazaki H, Iwano S, Kiyotani K, Matsumura K, Honda G, Nakagawa K, Ishizaki T, Kamataki T. CYP3A5 contributes significantly to CYP3A-mediated drug oxidations in liver microsomes from Japanese subjects. Drug Metab Pharmacokinet 2004; 19: 120-9.

7 deWildt SN, Kearns GL, Leeder JS, vandenAnker JN. Glucuronidation in humans -pharmacogenetic and developmental aspects. Clin Pharmacokinet 1999; 36: 439-52.

8 Daly AK. Significance of the minor cytochrome P450 3A isoforms. Clin Pharmacokinet 2006; 45: 13-31.

9 Koltin Y, Hitchcock CA. The search for new triazole antifungal agents. Curr Opin Chem Biol 1997; 1: 176-82.

10 Ito K, Iwatsubo T, Kanamitsu S, Ueda K, Suzuki H, Sugiyama Y. Prediction of pharmacokinetic alterations caused by drug-drug interactions: metabolic interaction in the liver. Pharmacol Rev 1998; 50: 387-411.

11 Ito K, Brown HS, Houston JB. Database analyses for the prediction of in vivo drug-drug interactions from in vitro data. Br J Clin Pharmacol 2004; 57: 473-86.

12 Niwa T, Murayama N, Emoto C, Yamazaki H. Comparison of kinetic parameters for drug oxidation rates and substrate inhibition potential mediated by cytochrome P450 3A4 and 3A5. Curr Drug Metab 2008; 9: 20-33.

13 Niwa T, Murayama N, Yamazaki H. Heterotropic cooperativity in oxidation mediated by cytochrome P450. Curr Drug Metab 2008; 9: 453-62.
14 Okada Y, Murayama N, Yanagida C, Shimizu M, Guengerich FP, Yamazaki H. Drug interactions of thalidomide with midazolam and cyclosporine A: heterotropic cooperativity of human cytochrome P450 3A5. Drug Metab Dispos 2009; 37: 18-23.

15 Ito K, Chiba K, Horikawa M, Ishigami M, Mizuno N, Aoki J, Gotoh Y, Iwatsubo T, Kanamitsu S, Kato M, Kawahara I, Niinuma K, Nishino A, Sato N, Tsukamoto Y, Ueda K, Itoh T, Sugiyama $Y$. Which concentration of the inhibitor should be used to predict in vivo drug interactions from in vitro data? AAPS PharmSci 2002; 4: E25.

16 Murayama N, Imai N, Nakane T, Shimizu M, Yamazaki H. Roles of CYP3A4 and CYP2C19 in methylhydroxylated and $\mathrm{N}$-oxidized metabolite formation from voriconazole, a new anti-fungal agent, in human liver microsomes. Biochem Pharmacol 2007; 73: 2020-6.

17 Gibbs MA, Thummel KE, Shen DD, Kunze KL. Inhibition of cytochrome P-450 3A (CYP3A) in human intestinal and liver microsomes: comparison of $K$ i values and impact of CYP3A5 expression. Drug Metab Dispos 1999; 27: 180-7.

18 Isoherranen N, Ludington SR, Givens RC, Lamba JK, Pusek SN, Dees EC, Blough DK, Iwanaga K, Hawke RL, Schuetz EG, Watkins PB, Thummel KE, Paine MF. The influence of CYP3A5 expression on the extent of hepatic CYP3A inhibition is substrate dependent: an in vitro-in vivo evaluation. Drug Metab Dispos 2008; 36: 146-54.

19 Obach RS, Walsky RL, Venkatakrishnan K, Gaman EA, Houston JB, Tremaine LM. The utility of in vitro cytochrome P450 inhibition data in the prediction of drug-drug interactions. J Pharmacol Exp Ther 2006; 316: 336-48.

20 Galetin A, Ito K, Hallifax D, Houston JB. CYP3A4 substrate selection and substitution in the prediction of potential drug-drug interactions. J Pharmacol Exp Ther 2005; 314: 180-90.

21 Racha JK, Zhao ZS, Olejnik N, Warner N, Chan R, Moore D, Satoh H. Substrate dependent inhibition profiles of fourteen drugs on CYP3A4 activity measured by a high throughput LCMS/MS method with four probe drugs, midazolam, testosterone, nifedipine and terfenadine. Drug Metab Pharmacokinet 2003; 18: 128-38.

22 Isoherranen N, Kunze KL, Allen KE, Nelson WL, Thummel KE. Role of itraconazole metabolites in CYP3A4 inhibition. Drug Metab Dispos 2004; 32: 1121-31.

23 Turpeinen M, Uusitalo J, Jalonen J, Pelkonen O. Multiple P450 substrates in a single run: rapid and comprehensive in vitro interaction assay. Eur J Pharm Sci 2005; 24: 123-32.

24 Di L, Kerns EH, Li SQ, Carter GT. Comparison of cytochrome P450 inhibition assays for drug discovery using human liver microsomes with LC-MS, rhCYP450 isozymes with fluorescence, and double cocktail with LC-MS. Int J Pharm 2007; 335: 1-11.

25 Venkataramanan R, Zang S, Gayowski T, Singh N. Voriconazole inhibition of the metabolism of tacrolimus in a liver transplant recipient and in human liver microsomes. Antimicrob Agents Chemother 2002; 46: 3091-3. 